Archaeologies: Journal of the World Archaeological Congress (ㄷ 2021)

https://doi.org/10.1007/s11759-021-09423-0

\title{
Afes WAC Inter-congress: WAC-9 Virtual WAC Pre-congress
}

\section{Dear WAC Members, Dear Colleagues,}

As the Covid-19 Pandemic is still limiting our communication and WAC-9 had to be postponed to 2022, we are going to organize an online pre-congress that may help us to keep contact and discuss the key issues of World Archaeology prior to the regular meeting in Prague 2022.

Please find below some technical information about the project that is currently in preparation by the WAC-9 LOC and the Guarant International Company.

\section{Pre-congress Description}

Within a week/two to organize 4-5 discussions open free of charge to members and supporters of WAC.

- There will always be one keynote followed by four papers and open discussion.

- Abstracts for individual sessions and contributions will be announced on the WAC-9 official website: https://www.wac-9.org/.

- Discussions will be held from several locations in local time zones: New Zealand-Czech Republic-USA...

- WAC would primarily care about the platform and backup of keynotes.

Each session consist of:

Keynote There will always be one keynote lecture pre-recorded as a backup in case of technical problems.

Participants It is estimated about 500-1000 participants each per webinar.

Lecture The lectures will take place in one piece without interference; discussion will take place after the lecture.

Login and timing Login details will be distributed upon request by email; timing of individual sessions will be adjusted to the local time zones of the individual virtual venues. 


\section{Solution and Design}

\section{Operation and Organization}

- A separate virtual room-webinar-will be created for each "open discussion".

- The webinar can accommodate up to 1000 participants at a time.

- All potential participants will be sent an invitation to register.

- With each keynote speaker we can complete about $1 \mathrm{~h}$ of training to pre-record a webinar.

- Within the webinar, there will always be one stream coordinator (director, support for speakers) and one technical support for participants.

\section{Technical Support}

- The conference will be provided using the CISCO Webex Platform, which will serve as a signpost for registration for specific webinars.

- Participants will receive an invitation with a link to the program, where participants will be able to register for individual webinars of their choice.

- The invitations will be sent to WAC members or the registration will be available as an URL link, on WAC website.

- The Webex platform has its own system of rights and roles for participants.

- Rights and roles can be set before and adjusted during the webinar.

- Separate room for independent chat and social events will be provided.

\section{Interactive Elements}

- For discussions, the platform has an integrated "Raise hand" function.

- During the webinar, participants can send questions to speakers/panellists thanks to the integrated Q\&A (questions and answers) function. This feature can be enabled/disabled. 
- During the webinar, participants will be able to chat with each other thanks to the integrated chat feature. This feature can be enabled/disabled

Yours Sincerely Jan Turek,

WAC-9 Academic Secretary

Publisher's Note Springer Nature remains neutral with regard to jurisdictional claims in published maps and institutional affiliations. 